\title{
Optimizations for the Computation of Radiative Corrections
}

\author{
Thomas Hahn ${ }^{\mathrm{a}}$ \\ ${ }^{a}$ Max-Planck-Institut für Physik \\ Föhringer Ring 6 \\ D-80805 Munich, Germany
}

MPI-PhT/2002-46

Two methods are presented with which the CPU time spent on the calculation of radiative corrections can be significantly reduced. The first is the parallelization of the program, which can be surprisingly simple to implement under certain circumstances often met in the calculation of radiative corrections. The second is the efficient direct calculation of fermion chains. The latter not only improves the overall performance of the program, but introduces better conceptual clarity as well, as it allows for a homogeneous treatment of bosonic and fermionic amplitudes.

\section{Introduction}

The calculation of radiative corrections is in general a very CPU-time consuming business, especially if "aggravating conditions" are met, such as the scans over large areas in parameter space typical for extensions of the Standard Model.

The running time of a program that computes radiative corrections can be written as

$$
T=N \tau+\varepsilon,
$$

where $N$ is the number of points in phase and parameter space and $\tau$ is the time to compute one such point. The remainder $\varepsilon$ accounts for all code which is executed only $n \ll N$ times, like initializations of model parameters, etc., and is thus generally negligible. Typical numbers are $N \sim 10^{5} \ldots 10^{7}$ and $\tau \sim 100 \mathrm{~ms}$.

The two techniques presented in this contribution reduce CPU time via both factors:

- Parallelization of the program reduces execution time from $\mathcal{O}(N)$ to $\mathcal{O}\left(N / N_{\text {processors }}\right)$,

- Directly computing fermion chains (in processes involving external fermions), rather than using the standard trace technique, reduces $\tau$ from $\mathcal{O}\left(n_{F}^{2}\right)$ to $\mathcal{O}\left(n_{F}\right)$, where $n_{F}$ is the number of fermionic structures.

The following discussion is limited to numerical programs. The examples are written in Fortran 77 , which is not only still in wide use among numerical programmers, but moreover constitutes a "lowest common denominator" in the sense that if a technique can be implemented in Fortran 77, it can be implemented in almost any language.

\section{Parallelization}

Parallelization in general is a very difficult topic, and considerable effort has been spent on the development of sophisticated libraries, compilers, and other tools. In the business of computing radiative corrections, however, programs often contain "naturally" parallel structures, for example if the same calculation is performed for different values of a parameter, viz.

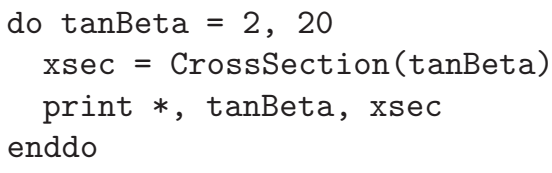

Such programs are known as essentially parallel, as each cycle of the loop may be executed independently without having to rearrange any code. In contrast, a parallelizing compiler will generally have to apply advanced techniques such as loop interchange and cache tiling before arriving at parallelizable code (see e.g. [1]).

The parallelization of such loops turns out to be surprisingly simple on a symmetric multiprocessing (SMP) architecture, that is, a single machine 
with several processors. Driven by demand in areas such as commercial Web hosting, SMP machines have meanwhile become affordable even for cash-strapped institutes and are well supported by all major operating systems.

The two basic operations are fork and wait: A fork creates an exact copy of the process that calls it; it returns 0 to the child process and the child's process id to the parent. The opposite is wait, which suspends the caller until one of its child processes has terminated.

Although fork and wait are not strictly part of the ANSI Fortran-77 Standard, they are available on many Unix systems. [1] They belong to a library of wrapper functions that provide Fortran access to certain functions of the $\mathrm{C}$ library. These functions are sometimes known also as the " $3 \mathrm{~F}$ " functions because they are described in section $3 \mathrm{~F}$ of the Unix man pages.

The implementation is straightforward: The parent process executes the main loop and in each cycle of the loop forks off a child process to do the actual calculation. The concurrent processes are automatically sent on the available processors by the operating system.

The reason why the implementation is so simple is that the forked processes run completely independently. This means that except for screen (and possibly file) output there are no resourcesharing issues to take care of, like avoiding simultaneous write access to some variable. By the same token, there is no simple way (in pure Fortran [1) for the child process to communicate its results back to the parent. If the results only have to be stored in a file, however, such problems can simply be solved by opening a unique output file for each child process and redirecting

\footnotetext{
${ }^{1}$ One notable exception is the g77 compiler. It is not difficult to work around this, however: set up a short C program, wrapper.c, which contains

\#include <unistd.h> with named pipes. 2) Data exchange via Unix domain sockets, although this requires some minor mixed-language programming in $\mathrm{C}$.
}

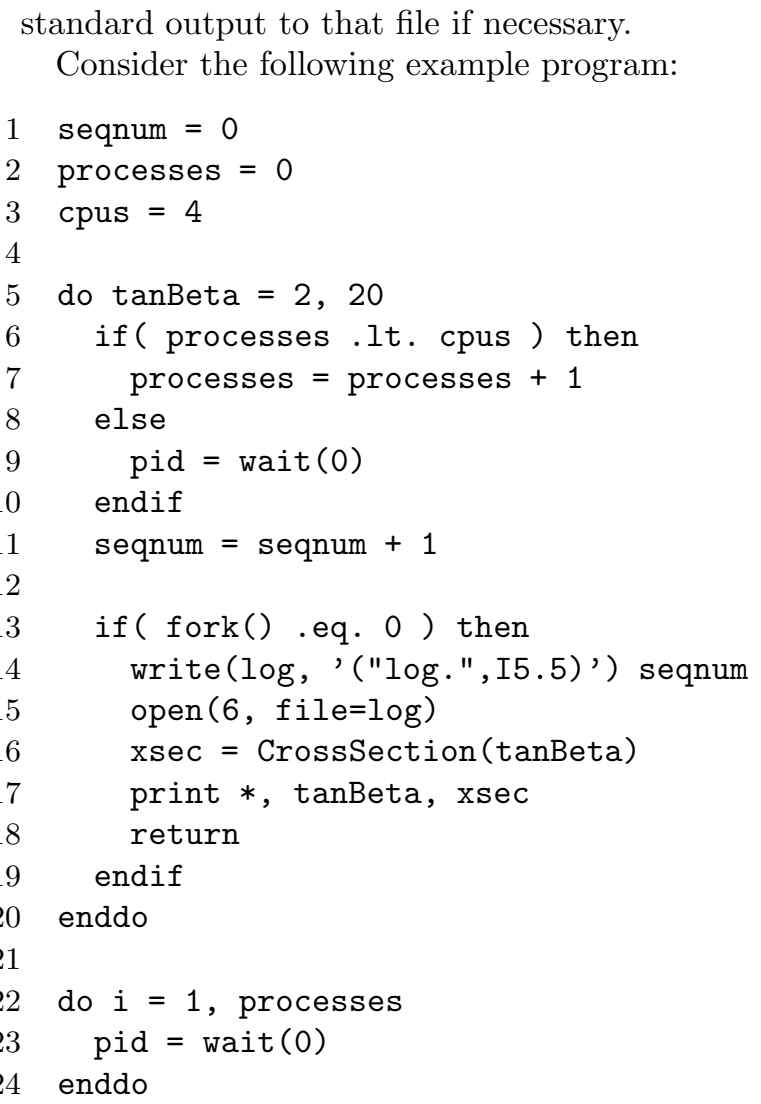

The main do loop ( $\ell .5-20)$ has been extended with respect to the serial version and falls into two blocks. The first block ( $\ell .6-11)$ is executed by the parent only. The parent calls fork in $\ell .13$ and immediately skips to the next cycle of the loop. The statements inside the if block ( $\ell$. 1418) are executed by the child process only.

The parent takes care not to spawn more child processes than there are processors available, so as not to overload the system. The number of concurrently running child processes is stored in the variable processes and the number of processors in cpus. The first cpus cycles of the loop "fill up" the available processors with jobs. After that the parent waits for a running child process to finish before proceeding to the next fork ( $\ell .9)$.

The child process, for which fork returns 0 , branches into the if block. It first generates a unique file name using a sequence number $(\ell .14)$ which it then opens as unit 6 , thus redirecting terminal output to this file. In this way it is guar- 
anteed that the output of concurrent processes is not mixed up. Most time is spent on the actual calculation ( $\ell .16-17)$ which is identical to the serial version. Unlike in the serial version, however, the child process does not return to the main loop, but terminates at the return statement ( $\ell .18)$.

After completing the main loop, the parent has to wait until all child processes have finished

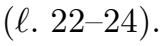

The overhead incurred by a fork is negligible unless very short units are parallelized. fork is a very common operation on preemptive multitasking systems such as Unix and is therefore usually implemented very efficiently. The Linux kernel, for instance, uses copy-on-write memory pages, that is, a memory page is not duplicated until either parent or child write on it. In fact, it will be difficult to notice any performance penalty of the parallel version running on a single CPU compared to the serial version.

If care is taken not to overload the system with more child processes than there are processors available (which would only require unnecessary task switches and use up memory resources) it is reasonable to expect a near-optimal speed-up, i.e. $T \rightarrow T / N$ on $N$ processors.

\section{Fermionic Objects}

Amplitudes of processes with external fermions can be written as

$$
\mathcal{M}=\sum_{i=1}^{n_{F}} c_{i} F_{i}
$$

where the $F_{i}$ are (products of) fermion chains, i.e. are of the form $\prod\left\langle u\left|\Gamma_{i}\right| v\right\rangle$, where $u$ and $v$ are spinors, and $\Gamma_{i}$ is a product of Dirac matrices.

One usually proceeds to compute probabilities, e.g. $|\mathcal{M}|^{2}$, rather than the amplitude $\mathcal{M}$ itself, because one can then use the trace technique:

$$
|\mathcal{M}|^{2}=\sum_{i, j=1}^{n_{F}} c_{i}^{*} c_{j} F_{i}^{*} F_{j}
$$

where $F_{i}^{*} F_{j}$ is computed as

$$
\begin{aligned}
F_{i}^{*} F_{j} & =\left\langle v\left|\bar{\Gamma}_{i}\right| u\right\rangle\left\langle u\left|\Gamma_{j}\right| v\right\rangle \\
& =\operatorname{Tr}\left(\bar{\Gamma}_{i}|u\rangle\left\langle u\left|\Gamma_{j}\right| v\right\rangle\langle v|\right) .
\end{aligned}
$$

The advantage is that no explicit representation of the spinors is needed since the projection operators can be expressed through Dirac matrices only, e.g. $\left|u_{\lambda}\right\rangle\left\langle u_{\lambda}\right|=\frac{1}{2}\left(\mathbb{1}+\lambda \gamma_{5}\right) \not p$ for a massless fermion.

The problem with the trace technique is that it scales as $n_{F}^{2}$. That is, one needs to compute all $n_{F}^{2}$ combinations of $F_{i}^{*} F_{j}$ and not just $n_{F}$ of the $F_{i}$ as for $\mathcal{M}$ alone. For $n_{F}=20$, for example, one has to compute 400 traces. More severely, matters get worse the more vectors are in the game, e.g. in multi-particle final states, or with polarization effects, because generally all combinations of vectors can appear in the $\Gamma_{i}$, and thus $n_{F} \sim$ (number of vectors)!.

The obvious alternative to the trace technique is to insert the 4-dimensional representation of the spinors and the Dirac matrices and work out the matrix algebra. This procedure is not entirely straightforward to implement in a language like Fortran, however, because in general Lorentz indices connect different fermion chains. Also, the calculational efficiency is not quite optimal because the 4-dim. representation contains redundancy, e.g. entire $2 \times 2$ blocks in the Dirac matrices are zero.

Actually, the 4-dim. representation is used for mathematical convenience only and, from the physical point of view, fermions are indeed more naturally represented by 2 -dim. objects which come in two kinds, left- and right-handed. This makes the Weyl representation an obvious choice.

The Weyl representation can in principle be used from the very beginning, i.e. already at the level of the Feynman rules [2]. This is only somewhat problematic for loop calculations, where regularization is needed, since it is not obvious how to extend the 2-dim. objects appearing in the Feynman rules to $D$ dimensions. In the present approach therefore everything is kept 4 (or $D$ ) dimensional during the algebraic simplification, such that e.g. $\gamma_{\mu} \gamma^{\mu} \rightarrow D$ can be replaced, and the Weyl representation is inserted only at the very end, just before the numerical evaluation, and only for objects involving external fermions (i.e. not for internal fermion traces).

The Dirac matrices are given in the Weyl rep- 
resentation by

$$
\begin{aligned}
& \gamma_{\mu}=\left(\begin{array}{cc}
0 & \sigma_{\mu} \\
\bar{\sigma}_{\mu} & 0
\end{array}\right), \omega_{+}=\left(\begin{array}{ll}
\mathbb{1} & 0 \\
0 & 0
\end{array}\right), \\
& \omega_{-}=\left(\begin{array}{ll}
0 & 0 \\
0 & \mathbb{1}
\end{array}\right),
\end{aligned}
$$

where the $\left(\bar{\sigma}_{\mu}\right)$ matrices are defined in terms of the ordinary Pauli matrices $\vec{\sigma}=\left(\sigma_{1}, \sigma_{2}, \sigma_{3}\right)$ as

$$
\sigma_{\mu}=(\mathbb{1},-\vec{\sigma}), \quad \bar{\sigma}_{\mu}=(\mathbb{1},+\vec{\sigma}) .
$$

Introducing furthermore 2-dim. spinors via

$$
\langle u|=\left(\left\langle u_{+}\left|,\left\langle u_{-}\right|\right), \quad \mid v\right\rangle=\left(\begin{array}{l}
\left|v_{-}\right\rangle \\
\left|v_{+}\right\rangle
\end{array}\right),\right.
$$

it is a simple exercise to show that every chiral 4-dim. Dirac chain can be converted to a single 2-dim. sigma chain:

$$
\begin{aligned}
& \left\langle u\left|\omega_{-} \gamma_{\mu} \gamma_{\nu} \cdots\right| v\right\rangle=\left\langle u_{-}\left|\bar{\sigma}_{\mu} \sigma_{\nu} \cdots\right| v_{ \pm}\right\rangle \\
& \left\langle u\left|\omega_{+} \gamma_{\mu} \gamma_{\nu} \cdots\right| v\right\rangle=\left\langle u_{+}\left|\sigma_{\mu} \bar{\sigma}_{\nu} \cdots\right| v_{\mp}\right\rangle
\end{aligned}
$$

That is, going from the 4-dim. to the 2-dim. representation does not increase $n_{F}$.

The two most important identities satisfied by the $(\bar{\sigma})$ matrices are the Fierz identities [ 3$]$

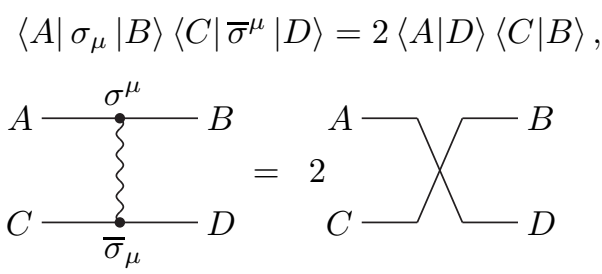

$$
\begin{aligned}
& \left\langle A\left|\bar{\sigma}_{\mu}\right| B\right\rangle\left\langle C\left|\bar{\sigma}^{\mu}\right| D\right\rangle=2\langle A|\varepsilon| C\rangle\langle B|\varepsilon| D\rangle,
\end{aligned}
$$

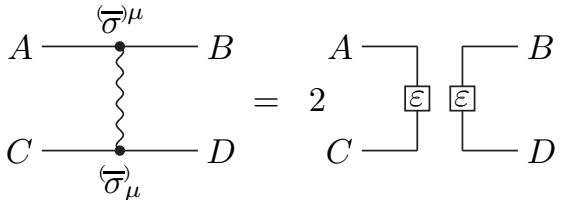

where $\varepsilon=\left(\begin{array}{cc}0 & 1 \\ -1 & 0\end{array}\right)$ is the spinor metric.

These identities allow to completely remove all Lorentz contractions between sigma chains. For the implementation in a computer program this is a decisive simplification, for it means that after application of the Fierz identities one can calculate one sigma chain at a time, independent of any other sigma chains.

To make the implementation even simpler, one can express also the remaining kinematical objects through $(\bar{\sigma})$ according to

$$
\begin{aligned}
g_{\mu \nu}= & \frac{1}{2} \operatorname{Tr}\left(\sigma_{\mu} \bar{\sigma}_{\nu}\right) \\
\varepsilon_{\lambda \mu \nu \rho}= & \frac{1}{2} \operatorname{Tr}\left(\sigma_{\lambda} \bar{\sigma}_{\mu} \sigma_{\nu} \bar{\sigma}_{\rho}\right)-g_{\lambda \mu} g_{\nu \rho} \\
& \quad+g_{\lambda \nu} g_{\mu \rho}-g_{\lambda \rho} g_{\mu \nu}
\end{aligned}
$$

Now the four-vectors themselves are no longer needed, but only their contractions with $(\bar{\sigma}$,

$$
\begin{aligned}
& \sigma_{\mu} k^{\mu}=\left(\begin{array}{cc}
k^{0}+k^{3} & k^{1}-\mathrm{i} k^{2} \\
k^{1}+\mathrm{i} k^{2} & k^{0}-k^{3}
\end{array}\right)=: \hat{k}, \\
& \bar{\sigma}_{\mu} k^{\mu}=\left(\begin{array}{cc}
k^{0}-k^{3} & -k^{1}+\mathrm{i} k^{2} \\
-k^{1}-\mathrm{i} k^{2} & k^{0}+k^{3}
\end{array}\right)=: \hat{\bar{k}} .
\end{aligned}
$$

Altogether now, the following objects and operations need to be implemented:

- arrays: all spinors, all vectors:

$$
\begin{aligned}
& \left|u_{ \pm}\right\rangle \sim\left(\begin{array}{l}
u_{1} \\
u_{2}
\end{array}\right), \\
& \hat{k}, \hat{\bar{k}} \sim\left(\begin{array}{ll}
a & b \\
c & d
\end{array}\right) .
\end{aligned}
$$

- functions: spinor $\times$ spinor $(\mathrm{ss})$, matrix $\times$ spinor $(\mathrm{ms})$, matrix $\times$ matrix $(\mathrm{mm})$ :

$$
\begin{aligned}
&\langle u \mid v\rangle \sim\left(\begin{array}{ll}
u_{1} & u_{2}
\end{array}\right) \cdot\left(\begin{array}{l}
v_{1} \\
v_{2}
\end{array}\right), \\
& \hat{k}|v\rangle \sim\left(\begin{array}{ll}
a & b \\
c & d
\end{array}\right) \cdot\left(\begin{array}{l}
v_{1} \\
v_{2}
\end{array}\right), \\
& \hat{k}_{1} \hat{k}_{2} \sim\left(\begin{array}{ll}
a_{1} & b_{1} \\
c_{1} & d_{1}
\end{array}\right) \cdot\left(\begin{array}{ll}
a_{2} & b_{2} \\
c_{2} & d_{2}
\end{array}\right) .
\end{aligned}
$$

These almost trivial functions are sufficient to compute arbitrary sigma chains by repeated application, e.g.

$$
\begin{aligned}
& \left\langle u\left|\hat{k}_{1} \hat{\bar{k}}_{2} \hat{k}_{3}\right| v\right\rangle= \\
& \operatorname{ss}\left(u, \operatorname{ms}\left(\hat{k}_{1}, \operatorname{ms}\left(\hat{\bar{k}}_{2}, \operatorname{ms}\left(\hat{k}_{3}, v\right)\right)\right)\right) .
\end{aligned}
$$

Note that in order to nest the functions inside each other as above, the matrix-valued 
functions have to write their results to an intermediate array (a kind of accumulator register) and return the location of that array.

Finally, one should note that when the fermion chains are computed directly, the amplitude $\mathcal{M}$ becomes a handy complex number, rather than a "bunch of form factors," $\left\{c_{i} F_{i}\right\}$. That is, to compute for example $|\mathcal{M}|^{2}$, one does not need to sum up a list of numbers, but only take the square of a complex variable. In this sense fermionic amplitudes are now treated on the same footing as bosonic amplitudes. Furthermore, since the $c_{i}$ no longer have to be factored out in front of the $F_{i}$, the analytic expression for $\mathcal{M}$ may be rearranged freely to yield the most compact form.

\section{Summary}

Two methods of speeding up the calculation of radiative corrections have been demonstrated.

\section{Parallelization}

Programs for computing radiative corrections are often natural candidates for parallelization. It is not difficult to attain near-optimal speed-up, i.e. $T \rightarrow T / N$ on $N$ processors.

On SMP machines, it is very easy to parallelize Fortran programs with only few changes in the code. The two basic functions fork and wait are available by default in many Fortran libraries, and even if not, only a trivial wrapper program in $\mathrm{C}$ is needed.

\section{Direct calculation of fermion chains}

The number of fermionic structures that have to be computed can be reduced from $\mathcal{O}\left(n_{F}^{2}\right)$, with the trace technique, to $\mathcal{O}\left(n_{F}\right)$ when calculating the fermion chains directly. Moreover, with the latter approach one gets a simple complex number for the amplitude $\mathcal{M}$.

Using the Weyl representation, the computation of the fermion chains is broken down from a 4-dim. to a 2-dim. problem. The Fierz identities allow to completely disentangle fermion chains connected by Lorentz indices.

The implementation is simple, even more so if one systematically expresses all kinematic objects through sigma chains, also non-fermionic ones like $g_{\mu \nu}, \varepsilon_{\lambda \mu \nu \rho}, k_{\mu}$.

\section{REFERENCES}

1. PGI User's Guide, STMicroelectronics, Inc., 1998, http://www.pgroup.com/ppro_docs/ pgiws_ug/pgiug_.htm.

2. S. Dittmaier, hep-ph/9805445.

3. V.I. Borodulin, R.N. Rogalyov, and S.R. Slabospitsky, hep-ph/9507456. 